\title{
NeutroAlgebra is a Generalization of Partial Algebra
}

\author{
Florentin Smarandache \\ University of New Mexico \\ Math \& Science Division \\ 705 Gurley Ave., Gallup, NM 87301, USA
}

\begin{abstract}
In this paper we recall, improve, and extend several definitions, properties and applications of our previous 2019 research referred to NeutroAlgebras and AntiAlgebras (also called NeutroAlgebraic Structures and respectively AntiAlgebraic Structures).

Let $\langle\mathrm{A}\rangle$ be an item (concept, attribute, idea, proposition, theory, etc.). Through the process of neutrosphication, we split the nonempty space we work on into three regions \{two opposite ones corresponding to $\langle\mathrm{A}\rangle$ and $\langle$ antiA $\rangle$, and one corresponding to neutral (indeterminate) <neutA> (also denoted <neutroA>) between the opposites $\}$, which may or may not be disjoint - depending on the application, but they are exhaustive (their union equals the whole space).
\end{abstract}

A NeutroAlgebra is an algebra which has at least one NeutroOperation or one NeutroAxiom (axiom that is true for some elements, indeterminate for other elements, and false for the other elements).

A Partial Algebra is an algebra that has at least one Partial Operation, and all its Axioms are classical (i.e. axioms true for all elements).

Through a theorem we prove that NeutroAlgebra is a generalization of Partial Algebra, and we give examples of NeutroAlgebras that are not Partial Algebras. We also introduce the NeutroFunction (and NeutroOperation).

Keywords: neutrosophy, algebra, neutroalgebra, neutroFunction, neutroOperation, neutroAxiom

\section{Neutrosophication by Tri-Sectioning the Space}

Let $X$ be a given nonempty space (or simply set) included into a universe of discourse $U$.

Let $\langle A\rangle$ be an item (concept, attribute, idea, proposition, theory, etc.) defined on the set $X$. Through the process of neutrosphication, we split the set $X$ into three regions [two opposite ones $\langle A\rangle$ and $\langle$ antiA $\rangle$, and one neutral (indeterminate) $<$ neutroA $>$ between them], regions which may or may not be disjoint - depending on the application, but they are exhaustive (their union equals the whole space).

The region denoted just by $\langle A\rangle$ is formed by all set's elements where $\langle A\rangle$ is true $\{$ degree of truth $(T)\}$, the region denoted by $\langle$ antiA $>$ is formed by all set's elements where $\langle A\rangle$ is false \{degree of falsehood $(F)$ \}, and the region denoted by $\langle$ neutroA $\rangle$ is formed by all set's elements where $\langle A\rangle$ is indeterminate (neither true nor false) \{degree of indeterminacy $(I)\}$.

We further on work with the following $\langle A\rangle$ concepts: Function, Operation, Axiom, and $\underline{\text { Algebra. }}$

Therefore, by tri-sectioning the set $X$ with respect to each such $\langle A\rangle$ concept, we get the following neutrosophic triplets corresponding to $(\langle A\rangle$, $\langle$ NeutroA $\rangle$, $\langle$ AntiA $\rangle)$ :

$<$ Function, NeutroFunction, AntiFunction>, 


\section{<Operation, NeutroOperation, AntiOperation>, \\ <Axiom, NeutroAxiom, AntiAxiom>, \\ $<$ Algebra, NeutroAlgebra, AntiAlgebra>.}

A NeutroAlgebra is an algebra which has at least one NeutroOperation or one NeutroAxiom (axiom that is true for some elements, indeterminate for other elements, and false for other elements).

We have proposed for the first time the NeutroAlgebraic Structures (or NeutroAlgebras), and in general the NeutroStructures, in 2019 [1] and further on in 2020 [2].

The NeutroAlgebra is a generalization of Partial Algebra, which is an algebra that has at least one Partial Operation, while all its Axioms are totally true (classical axioms).

We recall the Boole's Partial Algebras and the Effect Algebras as particular cases of Partial Algebras, and by consequence as particular cases of NeutroAlgebras.

In comparison between the Partial Algebra and the NeutroAlgebra:

i) When the NeutroAlgebra has no NeutroAxiom, and no outer-defined operation, it coincides with the Partial Algebra.

ii) There are NeutroAlgebras that have no NeutroOperations, but have NeutroAxioms. These are different from Partial Algebras.

iii) And NeutroAlgebras that have both, NeutroOperations and NeutroAxioms.

These are different from Partial Algebras too.

All the above will be proved in the following.

\section{2-4. Partially Inner-Defined, Partially Outer-Defined, or Partially Indeterminate}

Let $\mathrm{U}$ be a nonempty universe of discourse, and $\mathrm{X}$ and $\mathrm{Y}$ be two nonempty subsets of $\mathrm{U}$.

Let's consider a function:

$$
f: X \rightarrow Y .
$$

Let $a \in X$ be an element. Then, there are three possibilities:

i) [Inner-defined, or Well-defined; corresponding in neutrosophy to Truth (T)]

$f(a) \in Y$;

ii) [Outer-defined; corresponding in neutrosophy to Falsehood (F)]

$f(a) \in U-Y$;

iii) [Indeterminacy; corresponding in neutrosophy to Indeterminate (I)]

a) $f(a)=$ indeterminacy;

\{i.e. the value of $f(a)$ does exist, but we do not know it exactly;

for example, $f(a)=c$ or $d$, we know that $f(a)$ may be equal to $c$ or $d$ (but we are not sure to which one);

or, another example, we only know that $f(a) \neq e$, where the previous $c, d, e \in U\}$;

$\beta$ ) $f(a)=$ undefined (i.e. the value of $f(a)$ is not defined, or it does not exist - as in Partial Function); undefined is considered part of indeterminacy;

d) $f($ indeterminacy) $\in U$, but we either do not know the indeterminacy at all, or we only partially know some information about it

\{for example we know that $f(a$ or $b$ or $c) \in U$, where $a, b, c \in X$, but we are not sure if the argument is either $a$, or $b$, or $c\}$;

$\delta)$ more general: $f($ indeterminacyl $)=$ indeterminacy2, where indeterminacyl is a vaguely known value in $X$ and indeterminacy2 is a vaguely known value in $U$;

$\varepsilon$ ) By the way, there are many types of indeterminacies, we only gave above some elementary examples.

Consequently we have: 


\section{5-7. Definitions of Total InnerFunction, Total OuterFunction, Total IndeterminateFunction, and Total UndefinedFunction}

i) If for any $x \in X$ one has $f(x) \in Y$ (inner-ness, or well-defined), then $f$ is called a Total InnerFunction (or classical Total Function, or in general Function).

ii) If for any $x \in X$ one has $f(x) \in U$-Y (outer-ness, or outer-defined), then $f$ is called a Total OuterFunction (or AntiFunction).

iii) If for any $x \in X$ one has either $f(x)=$ indeterminacy, or $f$ (indeterminacy) $\in U$, or $f($ indeterminacy 1$)=$ indeterminacy2, then $f$ is called a Total IndeterminateFunction.

$\{$ As a particular case of the Total IndeterminateFunction there is the Total UndefinedFunction: when for any $x \in X$ one has $f(x)=$ undefined. $\}$

\section{Definition of Partial Function}

In the previous literature $\{[3],[4]\}$, the Partial Function was defined as follows:

A function $f: X \rightarrow Y$ is called a Partial Function if it is well-defined for some elements in $X$, and undefined for all the other elements in $X$. Therefore, there exist some elements $a \in X$ such that $f(a) \in Y$ (well-defined), and for all other element $b \in X$ one has $f(b)=$ (is) undefined.

We extend the partial function to NeutroFunction in order to comprise all previous $i$ ) - iii) situations.

\section{Definition of NeutroFunction}

A function $f: X \rightarrow Y$ is called a NeutroFunction if it has elements in $X$ for which the function is well-defined \{degree of truth $(T)$ \}, elements in $X$ for which the function is indeterminate \{degree of indeterminacy $(I)$ \}, and elements in $X$ for which the function is outer-defined \{degree of falsehood $(F)\}$, where $T, I, F \in[0,1]$, with $(\mathrm{T}, \mathrm{I}, \mathrm{F}) \neq(1,0,0)$ that represents the (Total) Function, and $(\mathrm{T}, \mathrm{I}, \mathrm{F}) \neq(0,0,1)$ that represents the AntiFunction.

In this definition "neutro" stands for neutrosophic, which means the existence of outer-ness, or undefined-ness, unknown-ness, or indeterminacy in general.

A NeutroFunction is more general, and it may include all three previous situations: elements in $X$ for which the function $f$ is well-defined, elements in $X$ for which function $f$ is indeterminate (including function's undefined values), and elements in $X$ for which function $f$ is outer-defined.

We have formed the following neutrosophic triplet:

$<$ Function, NeutroFunction (that includes the Partial Function), AntiFunction>.

Therefore, according to the above definitions, we have the following:

\section{Classification of Functions}

i) (Classical) Function, which is a function well-defined for all the elements in its domain of definition.

ii) NeutroFunction, which is a function partially well-defined, partially indeterminate, and partially outer-defined on its domain of definition.

iii) AntiFunction, which is a function outer-defined for all the elements in its domain of definition.

\section{Example of NeutroFunction}

Let $U=\{1,2,3,4,5,6,7,8,9,10,11,12\}$ be a universe of discourse, and two of its nonempty subsets $X=\{1,2,3$, $4,5,6\}, Y=\{7,8,9,10,11,12\}$, and the function $f$ constructed as follows:

$f: X \rightarrow Y$ such that 
$f(1)=7 \in Y$ (well-defined);

$f(2)=8 \in U-Y$ (outer-defined);

$f(3)=$ undefined (doesn't exist);

$f(4)=9$ or 10 or 11 (it does exist, but we do not know it exactly), therefore $f(4)=$ indeterminate;

$f($ some number greater $\geq 5)=12$, \{i.e. it can be $f(5)=12$ or $f(6)=12$, we are not sure about $\}$, therefore $f($ indeterminate $)=12$.

Similarly we defined the NeutroOperation.

\section{Definition of NeutroOperation}

An $n$-ary (for integer $n \geq 1$ ) operation $\omega: X^{n} \rightarrow Y$ is called a NeutroOperation if it is has n-plets in $X^{n}$ for which the operation is well-defined \{degree of truth $(T)$ \}, n-plets in $X^{n}$ for which the operation is indeterminate \{degree of indeterminacy $(I)\}$, and $n$-plets in $X^{n}$ for which the operation is outer-defined \{degree of falsehood $\left.(F)\right\}$, where $T, I, F$ $\in[0,1]$, with $(T, I, F) \neq(1,0,0)$ that represents the n-ary (Total) Operation, and $(T, I, F) \neq(0,0,1)$ that represents the n-ary AntiOperation.

Again, in this definition "neutro" stands for neutrosophic, which means the existence of outer-ness, or undefined-ness, or unknown-ness, or indeterminacy in general.

A NeutroOperation is more general, and it may include all previous situations: elements in $X^{n}$ for which the operation $\omega$ is well-defined, elements for which operation $\omega$ is outer-defined, and elements for which operation $\omega$ is indeterminate (including undefined).

\section{Definition of AntiOperation}

An $n$-ary (for integer $n \geq 1$ ) operation $\omega: X^{n} \rightarrow Y$ is called AntiOperation if for all $n$-plets $\left(x_{1}, x_{2}, \ldots, x_{n}\right) \in X^{n}$ one has $\omega\left(x_{1}, x_{2}, \ldots, x_{n}\right) \in U-Y$.

We have formed the neutrosophic triplet: <Operation, NeutroOperation, AntiOperation〉.

Therefore, according to the above definitions, we have the following:

\section{Classification of Operations}

On a given set:

i) (Classical) Operation is an operation well-defined for all the set's elements.

ii) NeutroOperation is an operation partially well-defined, partially indeterminate, and partially outerdefined on the given set.

iii) AntiFunction is an operation outer-defined for all the set's elements.

Further, we define the NeutroHyperOperation.

\section{Definition of NeutroHyperOperation}

Similarly, an $n$-ary (for integer $n \geq 1$ ) hyperoperation $\omega: X^{n} \rightarrow P(Y)$ is called a NeutroHyperOperation if it is has $n$ plets in $X^{n}$ for which the operation is well-defined $\omega\left(a_{1}, a_{2}, \ldots, a_{n}\right) \in P(Y)\{$ degree of truth $(T)\}, n$-plets in $X^{n}$ for which the operation is indeterminate \{degree of indeterminacy $(I)\}$, and $n$-plets in $X^{n}$ for which the operation is outer-defined $\omega\left(a_{1}, a_{2}, \ldots, a_{n}\right) \notin P(Y)\{$ degree of falsehood $(F)\}$, where $T, I, F \in[0,1]$, with $(T, I, F) \neq(1,0,0)$ that represents the $\mathrm{n}$-ary (Total) HyperOperation, and $(T, I, F) \neq(0,0,1)$ that represents the n-ary AntiHyperOperation. 
Again, in this definition "neutro" stands for neutrosophic, which means the existence of outer-ness, or undefined-ness, or unknown-ness, or indeterminacy in general.

A NeutroOperation is more general, and it may include all previous situations: elements in $X^{n}$ for which the operation $\omega$ is well-defined, elements for which operation $\omega$ is outer-defined, and elements for which operation $\omega$ is indeterminate (including undefined).

\section{Definition of AntiHyperOperation}

An $n$-ary (for integer $n \geq 1$ ) operation $\omega: X^{n} \rightarrow P(Y)$ is called AntiHyperOperation if it is outer-defined for all the $n$ plets in $\mathrm{X}^{n}$. Or, for any $n$-plet $\left(x_{1}, x_{2}, \ldots, x_{n}\right) \in X^{n}$ one has $\omega\left(x_{1}, x_{2}, \ldots, x_{n}\right) \notin P(Y)$.

Again, we have formed a neutrosophic triplet:

$<$ HyperOperation, NeutroHyperOperation, AntiHyperOperation>.

Similarly, according to the above definitions, we have the following:

\section{Classification of HyperOperations}

On a given set:

i) (Classical) HyperOperation is a hyper-operation well-defined for all the set's elements.

ii) NeutroHyperOperation is a hyper-operation partially well-defined, partially indeterminate, and partially outer-defined on the given set.

iii) AntiHyperFunction is a hyper-operation outer-defined for all the set's elements.

\section{Definition of Universal Algebra}

In the previous literature there exist the following.

The (classical) Universal Algebra (or General Algebra) is a branch of mathematics that studies classes of (classical) algebraic structures.

\section{Definition of Algebraic Structure}

A (classical) Algebraic Structure (or Algebra) is a nonempty set A endowed with some (totally well-defined) operations (functions) on A, and satisfying some (classical) axioms (totally true) - according to the Universal Algebra.

\section{Definition of Partial Algebra}

A (classical) Partial Algebra is an algebra defined on a nonempty set PA that is endowed with some partial operations (or partial functions: partially well-defined, and partially undefined). While the axioms (laws) defined on a Partial Algebra are all totally $(100 \%)$ true.

\section{Definition of Effect Algebra}

A set $\mathrm{L}$ that contains two special elements $0, l \in L$, and endowed with a partially defined binary operation $\bigoplus$ that satisfies the following conditions (Foulis and Bennett [4]).

For all $p, q, r \in L$ one has:

i) If $p \oplus q$ is defined, then $q \oplus p$ is defined and $p \oplus q=q \oplus p$ [Commutativity].

ii) If $q \oplus r$ is defined and $p \oplus(q \oplus r)$ is defined, then $p \oplus q$ is defined and ( $\mathrm{p} \oplus q) \oplus \mathrm{r}$ is defined, and $p \oplus(q \oplus r)$ $=(\mathrm{p} \oplus q) \oplus \mathrm{r}$ [Associativity]. 
iii) For every $p \in L$ there exists a unique $q \in L$ such that $p \oplus q$ is defined and $p \oplus q=1$ (Orthosupplementation).

iv) If $l \oplus p$ is defined, then $p=O$ (Zero-One Law).

Clearly, the Effect Algebra is a particular case of Partial Algebra, since it has a partial operation $\oplus$, and all its (Commutative, Associative, Orthosupplementation, and Zero-One) Laws are totally true.

\section{Definition of Boole's Partial Algebras}

Let $\mathrm{U}$ be a universe of discourse, $\mathrm{Su}(\mathrm{U})$ the collection of subsets of $\mathrm{U}$, and $\mathrm{S}(\mathrm{U})$ the partial algebra $(\mathrm{Su}(\mathrm{U}),+,, ;,-, 0$, 1). Two partial operations ( + and - ) were defined by George Boole (Burris and Sankappanavar [5]):

$A+B:=A \cup B$, provided $A \cap B=\phi$, otherwise undefined;

and

$A-B:=A \backslash B$, provided $B \subseteq A$, otherwise undefined;

one total operation:

$A \cdot B=A \cap B$

and two constants:

$1:=U$

$0:=\phi$.

Obviously, Boole's Partial Algebras are partial algebras since they have at least one partial operation, while its axioms are totally true.

Now we extend the Partial Algebra to NeutroAlgebra, but first we recall the below.

\section{Classification of Axioms:}

i) A (classical) Axiom defined on a nonempty set is an axiom that is totally true (i.e. true for all set's elements).

ii) A NeutroAxiom (or Neutrosophic Axiom) defined on a nonempty set is an axiom that is true for some set's elements \{degree of truth $(T)$ \}, indeterminate for other set's elements \{degree of indeterminacy $(I)\}$, or false for the other set's elements \{degree of falsehood $(F)\}$, where $T, I, F \in[0, I]$, with $(T, I, F)$ $\neq(1,0,0)$ that represents the (classical) Axiom, and $(T, I, F) \neq(0,0,1)$ that represents the AntiAxiom.

iii) An AntiAxiom defined on a nonempty set is an axiom that is false for all set's elements.

Therefore, we have formed the neutrosophic triplet: 〈Axiom, NeutroAxiom, AntiAxiom〉.

\section{Classification of Algebras}

i) A (classical) Algebra is a nonempty set CA that is endowed with total operations (or total functions, i.e. true for all set's elements) and (classical) Axioms (also true for all set's elements).

ii) A NeutroAlgebra (or NeutroAlgebraic Structure) is a nonempty set NA that is endowed with: at least one NeutroOperation (or NeutroFunction), or one NeutroAxiom that is referred to the set's (partial-, neutro, or total-) operations.

iii) An AntiAlgebra (or AntiAlgebraic Structure) is a nonempty set AA that is endowed with at least one AntiOperation (or AntiFunction) or at least one AntiAxiom.

Therefore, we have formed the neutrosophic triplet: 
$<$ Algebra, NeutroAlgebra (which includes the Partial Algebra), AntiAlgebra>.

\section{Definition of Universal NeutroAlgebra}

The Universal NeutroAlgebra (or General NeutroAlgebra) is a branch of neutrosophic mathematics that studies classes of NeutroAlgebras and AntiAlgebras.

\section{Applications of NeutroFunctions and NeutroAlgebras}

Applicability of Partial Functions, when the domain is not well-known, are in computer science, computability theory, programming language, real analysis, complex analysis, charts in the atlases, recursion theory, category theory, etc.

NeutroFunctions (NeutroOperations), when the domain and/or range are/is not well-known, have a larger applicable field since, besides Partial Functions' undefined values, NeutroFunctions include functions' outer-defined and/or indeterminate values referred not only to the functions' not-well-known domain but to the functions' not-well-known range too.

NeutroAlgebras, in addition to NeutroFunctions, is equipped with NeutroAxioms that better reflect our reality where not all individuals totally agree or totally disagree with some regulation (law, rule, action, organization, idea, etc.), but each individual expresses partial degree of approval, partial degree of ignorance, and partial degree of disapproval of the regulation. NeutroAxioms are true for some elements, indeterminate for others, and false for other elements.

\section{NeutroAxioms in our World}

Unlike the idealistic or abstract algebraic structures, from pure mathematics, constructed on a given perfect space (set), where the axioms (laws, rules, theorems, results etc.) are totally (100\%) true for all space's elements, our World and Reality consist of approximations, imperfections, vagueness, and partialities.

Most of mathematical models are too rigid to completely describe the imperfect reality. Many axioms are actually NeutroAxioms (i.e. axioms that are true for some space's elements, indeterminate for other space's elements, and false for other space's elements). See below several examples.

In Soft Sciences [2] the laws are interpreted and re-interpreted; in social, political, religious legislation the laws are flexible; the same law may be true from a point of view, and indeterminate or false from another point of view. Thus the law is partially true and partially indeterminate (neutral) or false (it is a neutrosophic law, or NeutroLaw).

Many interpretations have a degree of objectivity, a degree of neutrality (indeterminacy), and a degree of subjectivity. The cultural, tradition, religious, and psychological factors play important roles in interpretations and actions for or against some regulations.

a) For example, "gun control". There are people supporting it because of too many crimes and violence (and they are right), and people that oppose it because they want to be able to defend themselves and their houses (and they are right too); there also are ignorant people who do not care (so, they do not manifest for or against it).

Besides ignorant (neutral) people, we see two opposite propositions, both of them true, but from different points of view (from different criteria/parameters; plithogenic logic may better be used herein, since the truth-value of a proposition is calculated from various points of view - obtaining different results). How to solve this? Going to the middle, in between opposites (as in neutrosophy): allow military, police, security, registered hunters to bear arms; prohibit mentally ill, sociopaths, criminals, violent people from bearing arms; and background check on everybody that buys arms, etc.

b) Similarly for "abortion". Some people argue that by abortion one kills a life (which is true), others support the idea of the woman to be master of her body (which is true as well), and again the category of ignorants.

c) A law applying for a category of people (degree of truth), but not applying for another category of people (degree of falsehood).

For example, in India a Hindi man is allowed to marry only one wife, while a Muslim man is allowed to marry up to four wives. 
d) Double Standard: a rule applying for some people, but not applying for other people that for example may have a higher social rank.

e) Hypocrisy: criticizing your enemies (but not your friends!) for what your friends do too!

Or praising your friends (but not your enemies!) for what your enemies do too!

That's why the NeutroAlgebras better model our imprecise reality and they are needed to be studied, since they are equipped with NeutroOperations (partially true, partially indeterminate, and partially false operations) and NeutroAxioms (partially true, partially indeterminate, and partially false axioms), all designed on a not-well-known space.

\section{Theorem 1}

The NeutroAlgebra is a generalization of Partial Algebra.

As a consequence, NeutroAlgebra is a generalization of Effect Algebra and of Boole's Partial Algebras.

Proof.

Since the Partial Algebra is equipped with partially defined operations, they are NeutroAlgebras according with the above definition of NeutroAlgebras. But the converse is not true.

Further on, the Effect Algebra and the Boole's Partial Algebras are particular cases of Partial Algebra, therefore particular cases of NeutroAlgebra.

\section{Example of NeutroAlgebra that is not a Partial Algebra}

Let the set $S=(0, \infty)$, endowed with the real division $\div$ of numbers. $(S, \div)$ is well defined, since

$\div$ is a total operation (there is no division by zero).

$S$ is NeutroAssociative, because, from $x, y, z \in S$ such that

$x \div(y \div z)=(x \div y) \div z$

one gets $\frac{x z}{y}=\frac{x}{y z}$ or $x y z^{2}=x y$ or $z^{2}=1$ (since both $x, y \neq 0$ ), whence $z=1$ (the other solution

$z=-1$ does not belong to $S$ ).

Therefore, $(S, \div)$ is: associative for the triplets of the form $\{(x, y, 1), x, y \in S\}$, while for other triplets $\{(x, y, z), x, y$, $z \in S$, and $z \neq 1\}$ it is not associative. So, $S$ is partially associative and partially nonassociative (that we call NeutroAssociative).

Thus $(S, \div)$ is a classical groupoid, it is neither a partial algebra nor an effect algebra since its operation $\div$ is not a partial operation (but a total operation), and it is a NeutroSemigroup (since it is well-defined and neutroassociative) which means part of the general NeutroAlgebra.

Thus we proved that there are NeutroAlgebras that are different from Partial Algebras.

\section{Other Examples of NeutroAlgebras vs. Partial Algebras}

Let $U=\{a, b, c\}$ be a universe of discourse and $S=\{a, b\}$ one of its nonempty subsets.

i) Structure $S_{1}=\left(S, *_{1}\right)$, constructed as below using Cayley Table:

\begin{tabular}{l|ll}
$*_{1}$ & $a$ & $b$ \\
\hline$a$ & $b$ & $a$ \\
$b$ & $a$ & undefined
\end{tabular}

$*_{l}$ is a partially defined operation since $b^{*} b=$ undefined, but for all $x \neq b$ or $y \neq b, x *_{l} y$ is defined. 
The axiom of commutativity is totally true, since $a^{*}{ }_{1} b$ and $b{ }^{*} a$ are defined, and they are equal: $a{ }_{1} b=a=b{ }_{1} a$. Therefore, $S_{l}$ equipped with the axiom of commutativity is a partial algebra.

But $S_{I}$ equipped with the axiom of associativity is not a partial algebra, since the associativity is partially true and partially indeterminate or partially false (i.e. NeutroAssociativity):

$a *_{1}\left(b *_{1} a\right)=a *_{1} a=b$, and $\left(a *_{1} b\right) *_{1} a=a *_{1} a=b \quad$ (degree of truth);

but $a *_{1}\left(a *_{1} b\right)=a *_{1} a=b$ while $\left(a *_{1} a\right){ }_{1} b=b{ }_{1} b=$ undefined $\neq b$ (degree of falsehood).

Therefore, $S_{l}$ equipped with the axiom of associativity is a Neutro-algebra.

ii) Structure $S_{2}=\left(S, *_{2}\right)$, constructed as below using Cayley Table:

\begin{tabular}{l|ll}
$*_{2}$ & $a$ & $b$ \\
\hline$a$ & $b$ & $a$ \\
$b$ & $a$ & $c \notin S$
\end{tabular}

$*_{2}$ is an outer-operation since $b_{2} b=c \in U-S$ is outer-defined, but for all $x \neq b$ or $y \neq b, x *_{2} y$ is inner-defined. Because $*_{2}$ is not partially defined (since $b *_{2} b \neq$ undefined), $S_{2}$ cannot be a partial algebra.

Similarly, the axiom of commutativity is totally true, since $a^{*} b$ and $b *_{2} a$ are defined, and they are equal: $a *_{2} b=a=$ $b *_{2} a$.

Therefore, $S_{2}$ equipped with the axiom of commutativity is an outer-algebra (which is a particular case of NeutroAlgebra).

But $S_{2}$ equipped with the axiom of associativity is not an outer-algebra, since the associativity is partially true and partially indeterminate or partially false (i.e. NeutroAssociativity):

$a *_{2}\left(b *_{2} a\right)=a *_{2} a=b$, and $\left(a *_{2} b\right) *_{2} a=a *_{2} a=b$ (degree of truth);

but $a *_{2}\left(a *_{2} b\right)=a_{2} *_{2} a=b$ while $\left(a *_{2} a\right) *_{2} b=b *_{2} b=c \neq b$ (degree of falsehood).

Therefore, $S_{2}$ equipped with the axiom of associativity is a Neutro-algebra.

iii) Structure $S_{3}=\left(S, *_{3}\right)$, constructed as below using Cayley Table:

\begin{tabular}{l|ll}
$*_{2}$ & $a$ & $b$ \\
\hline$a$ & $b$ & $a$ \\
$b$ & $a$ & $a$ or $b$
\end{tabular}

$*_{3}$ is an indeterminate-operation since $b^{*}{ }_{3} b=a$ or $b$ (indeterminate), but for all $x \neq b$ or $y \neq b, x_{3} y$ is well-defined. The same, because $*_{3}$ is not partially defined (since $b *_{3} b \neq$ undefined), $S_{3}$ cannot be a partial algebra.

Similarly, the axiom of commutativity is totally true, since $a^{*} b$ and $b *_{3} a$ are defined, and they are equal: $a *_{3} b=a=$ $b *_{3} a$.

Therefore, $S_{3}$ equipped with the axiom of commutativity is an indeterminate-algebra (a particular case of NeutroAlgebra). 
But $S_{3}$ equipped with the axiom of associativity is not an indeterminate-algebra, since the associativity is partially true and partially indeterminate or partially false (i.e. NeutroAssociativity):

$a *_{3}\left(b *_{3} a\right)=a *_{3} a=b$, and $\left(a *_{3} b\right) *_{3} a=a *_{3} a=b \quad$ (degree of truth);

but $a *_{3}\left(a *_{3} b\right)=a *_{3} a=b$ while $\left(a *_{3} a\right)_{3} b=b *_{3} b=(a$ or $b) \neq b$ (degree of falsehood).

Therefore, $S_{3}$ equipped with the axiom of associativity is a NeutroAlgebra.

\section{The main distinction between Partial Algebra vs. NeutroAlgebra}

A Partial Algebra has at least one Partial Operation, while all Axioms involving its partial and total operations (Associativity, Commutativity, etc.) are $100 \%$ true.

Whilst a NeutroAlgebra has at least one NeutroOperation (which is an extension of Partial Operation) or one NeutroAxiom:

i) When the NeutroAlgebra has no NeutroAxiom, it coincides with the Partial Algebra.

ii) There are NeutroAlgebras that have no NeutroOperations, but have NeutroAxioms. These are different from Partial Algebras.

iii) And NeutroAlgebras that have both, NeutroOperations and NeutroAxioms. Also, these are different from Partial Algebras.

\section{Remark 1}

For the study of NeutroAlgebras the names of axioms (to be taken into consideration if they are partially true, partially indeterminate, partially false) and similarly for the study of AntiAlgebras the names of axioms (to be taken into consideration if they are totally false) should from the beginning be specified - since many axioms may fall in such categories.

\section{Acknowledgement}

The author thanks Prof. Dr. Xiaohong Zhang from Shaanxi University of Science and Technology, Xi'an, China, and Dr. Said Broumi, from University of Hassan II, Casablanca, Morocco, for their comments on this paper.

\section{REFERENCES}

[1] Florentin Smarandache, Introduction to NeutroAlgebraic Structures and AntiAlgebraic Structures, in Advances of Standard and Nonstandard Neutrosophic Theories, Pons Publishing House Brussels, Belgium, Ch. 6, pp. 240-265, 2019.

[2] Florentin Smarandache, Introduction to NeutroAlgebraic Structures and AntiAlgebraic Structures (revisited), Neutrosophic Sets and Systems, vol. 31, pp. 1-16, 2020. DOI: 10.5281/zenodo.3638232.

[3] Horst Reichel, Structural induction on partial algebras, Akademie-Verlag, 1984.

[4] D. Foulis and M. Bennett. Effect algebras and unsharp quantum logics, Found. Phys., 24(10): 1331-1352, 1994. 
[5] Stanley N. Burris and H. P. Sankappanavar, The Horn's Theory of Boole's Partial Algebras, The Bulletin of Symbolic Logic, Vol. 19, No. 1, 97-105, 2013. 